\title{
REAL GEL'FAND-MAZUR DIVISION ALGEBRAS
}

\author{
MATI ABEL and OLGA PANOVA
}

\author{
Received 4 November 2002
}

\begin{abstract}
We show that the complexification $(\tilde{A}, \tilde{\tau})$ of a real locally pseudoconvex (locally absorbingly pseudoconvex, locally multiplicatively pseudoconvex, and exponentially galbed) algebra $(A, \tau)$ is a complex locally pseudoconvex (resp., locally absorbingly pseudoconvex, locally multiplicatively pseudoconvex, and exponentially galbed) algebra and all elements in the complexification $(\tilde{A}, \tilde{\tau})$ of a commutative real exponentially galbed algebra $(A, \tau)$ with bounded elements are bounded if the multiplication in $(A, \tau)$ is jointly continuous. We give conditions for a commutative strictly real topological division algebra to be a commutative real Gel'fand-Mazur division algebra.
\end{abstract}

2000 Mathematics Subject Classification: 46H05, 46H20.

1. Introduction. Let $\mathbb{K}$ be one of the fields $\mathbb{R}$ of real numbers or $\mathbb{C}$ of complex numbers. A topological algebra $A$ is a topological vector space over $\mathbb{K}$ in which the multiplication is separately continuous. Herewith, $A$ is called a real topological algebra if $\mathbb{K}=\mathbb{R}$ and a complex topological algebra if $\mathbb{K}=\mathbb{C}$. We classify topological algebras in a similar way as topological vector spaces. For example, a topological algebra $A$ is

(a) a Fréchet algebra if it is complete and metrizable;

(b) an exponentially galbed algebra (see [3, 13]) if its underlying topological vector space is exponentially galbed, that is, for each neighborhood $O$ of zero in $A$, there exists another neighborhood $U$ of zero such that

$$
\left\{\sum_{k=0}^{n} \frac{a_{k}}{2^{k}}: a_{0}, \ldots, a_{n} \in U\right\} \subset O
$$

for each $n \in \mathbb{N}$;

(c) a locally pseudoconvex algebra (see [5, 7]) if its underlying topological vector space is locally pseudoconvex, that is, $A$ has a base $\left\{U_{\alpha}, \alpha \in \mathscr{A}\right\}$ of neighborhoods of zero in which every set $U_{\alpha}$ is balanced (i.e., $\lambda U_{\alpha} \in U_{\alpha}$ whenever $|\lambda| \leqslant 1$ ) and pseudoconvex (i.e., $U_{\alpha}+U_{\alpha} \subset 2^{1 / k_{\alpha}} U_{\alpha}$ for some $\left.k_{\alpha} \in(0,1]\right)$. Herewith, every locally pseudoconvex algebra is an exponentially galbed algebra.

In particular, when $k_{\alpha}=k\left(k_{\alpha}=1\right)$ for each $\alpha \in \mathscr{A}$, then a locally pseudoconvex algebra $A$ is called a locally $k$-convex algebra (resp., locally convex 
algebra). It is well known (see [14, page 4]) that the topology of a locally pseudoconvex algebra $A$ can be given by means of a family $\mathscr{P}=\left\{p_{\alpha}: \alpha \in A\right\}$ of $k_{\alpha}$-homogeneous seminorms, where $k_{\alpha} \in(0,1]$ for each $\alpha \in A$. A locally pseudoconvex algebra is called a locally absorbingly pseudoconvex (shortly, locally A-pseudoconvex) algebra (see [5]) if every seminorm $p \in \mathscr{P}$ is A-multiplicative, that is, for each $a \in A$ there are positive numbers $M_{p}(a)$ and $N_{p}(a)$ such that

$$
p(a b) \leqslant M_{p}(a) p(b), \quad p(b a) \leqslant N_{p}(a) p(b),
$$

for each $b \in A$. In particular, when $M_{p}(a)=N_{p}(a)=p(a)$ for each $a \in A$ and $p \in \mathscr{P}$, then $A$ is called a locally multiplicatively pseudoconvex (shortly, locally m-pseudoconvex) algebra.

Moreover, a topological algebra $A$ over $\mathbb{K}$ with a unit element is a $Q$-algebra (see $[10,15,16]$ ) if the set of all invertible elements of $A$ is open in $A$ and a $Q$-algebra $A$ is a Waelbroeck algebra (see $[4,10]$ ) or a topological algebra with continuous inverse (see $[9,11]$ ) if the inversion $a \rightarrow a^{-1}$ in $A$ is continuous.

An element $a$ of a topological algebra $A$ is said to be bounded (see [6]) if for some nonzero complex number $\lambda_{a}$, the set

$$
\left\{\left(\frac{a}{\lambda_{a}}\right)^{n}: n \in \mathbb{N}\right\}
$$

is bounded in $A$. A topological algebra, in which all elements are bounded, will be called a topological algebra with bounded elements.

Let now $A$ be a topological algebra over $\mathbb{K}$ and $m(A)$ the set of all closed regular two-sided ideals of $A$, which are maximal as left or right ideals. In case when the quotient algebra $A / M$ (in the quotient topology) is topologically isomorphic to $\mathbb{K}$ for each $M \in m(A)$, then $A$ is called a Gel'fand-Mazur algebra (see $[1,4,2])$. Herewith, $A$ is a real Gel'fand-Mazur algebra if $\mathbb{K}=\mathbb{R}$ and a complex Gel'fand-Mazur algebra if $\mathbb{K}=\mathbb{C}$. Main classes of complex Gel'fandMazur algebras have been given in $[4,2,5]$. Several classes of real Gel'fandMazur division algebras are described in the present paper.

2. Complexification of real algebras. Let $A$ be a (not necessarily topological) real algebra and let $\tilde{A}=A+i A$ be the complexification of $A$. Then every element $\tilde{a}$ of $\tilde{A}$ is representable in the form $\tilde{a}=a+i b$, where $a, b \in A$ and $i^{2}=-1$. If the addition, scalar multiplication, and multiplication in $\tilde{A}$ are to be defined by

$$
\begin{aligned}
(a+i b)+(c+i d) & =(a+c)+i(b+d), \\
(\alpha+i \beta)(a+i b) & =(\alpha a-\beta b)+i(\alpha b+\beta a), \\
(a+i b)(c+i d) & =(a c-b d)+i(a d+b c),
\end{aligned}
$$

for all $a, b, c, d \in A$ and $\alpha, \beta \in \mathbb{R}$, then $\tilde{A}$ is a complex algebra with zero element $\theta_{\tilde{A}}=\theta_{A}+i \theta_{A}$ (here and later on $\theta_{A}$ denotes the zero element of $A$ ). In case 
when $A$ has the unit element $e_{A}$, then $e_{\tilde{A}}=e_{A}+i \theta_{A}$ is the unit element of $\tilde{A}$. Herewith, $\tilde{A}$ is an associative (commutative) algebra if $A$ is an associative (resp., commutative) algebra. Therefore, we can consider $A$ as a real subalgebra of $\tilde{A}$ under the imbedding $v$ from $A$ into $\tilde{A}$ defined by $v(a)=a+i \theta_{A}$ for each $a \in A$.

A real (not necessarily topological) algebra $A$ is called a formally real algebra if from $a, b \in A$ and $a^{2}+b^{2}=\theta_{A}$ that follows that $a=b=\theta_{A}$ and is called a strictly real algebra if $\operatorname{sp}_{\tilde{A}}\left(a+i \theta_{A}\right) \subset \mathbb{R}$ (here $\operatorname{sp}_{A}(a)$ denotes the spectrum of $a \in A$ in $A$ ). It is known (see, e.g., [7, Proposition 1.9.14]) that every formally real division algebra is strictly real and every commutative strictly real division algebra is formally real.

Let now $(A, \tau)$ be a real topological algebra and $\left\{U_{\alpha}: \alpha \in \mathscr{A}\right\}$ a base of neighborhoods of zero of $(A, \tau)$. As usual (see [7, 17]), we endow $\tilde{A}$ with the topology $\tilde{\tau}$ in which $\left\{U_{\alpha}+i U_{\alpha}: \alpha \in \mathscr{A}\right\}$ is a base of neighborhoods of zero. It is easy to see that $(\tilde{A}, \tilde{\tau})$ is a topological algebra and the multiplication in $(\tilde{A}, \tilde{\tau})$ is jointly continuous if the multiplication in $(A, \tau)$ is jointly continuous (see [7, Proposition 2.2.10]). Moreover, the underlying topological space of $(\tilde{A}, \tilde{\tau})$ is a Hausdorff space if the underlying topological space of $(A, \tau)$ is a Hausdorff space.

3. Complexification of real locally pseudoconvex algebras. Let $(A, \tau)$ be a real locally pseudoconvex algebra and $\left\{p_{\alpha}: \alpha \in \mathscr{A}\right\}$ a family of $k_{\alpha}$-homogeneous seminorms on $A$ (where $k_{\alpha} \in(0,1]$ for each $\alpha \in \mathscr{A}$ ), which defines the topology $\tau$ on $A$ and $\tilde{A}$, the complexification of $A$,

$$
\begin{aligned}
& \Gamma_{k_{\alpha}}\left(U_{\alpha}+i \theta_{A}\right) \\
&=\left\{\sum_{k=1}^{n} \lambda_{k}\left(u_{k}+i \theta_{A}\right): n \in \mathbb{N}, u_{1}, \ldots, u_{n} \in U_{\alpha}, \lambda_{1}, \ldots, \lambda_{n} \in \mathbb{C} \text { and } \sum_{k=1}^{n}\left|\lambda_{k}\right|^{k_{\alpha}} \leqslant 1\right\}, \\
& q_{\alpha}(a+i b)=\inf \left\{|\lambda|^{k_{\alpha}}:(a+i b) \in \lambda \Gamma_{k_{\alpha}}\left(U_{\alpha}+i \theta_{A}\right)\right\}
\end{aligned}
$$

for each $a+i b \in \tilde{A}$. Then $\Gamma_{k_{\alpha}}\left(U_{\alpha}+i \theta_{A}\right)$ is the absolutely $k_{\alpha}$-convex hull of $U_{\alpha}+i \theta_{A}$ for each $\alpha \in \mathscr{A}$ and $q_{\alpha}$ is a $k_{\alpha}$-homogeneous Minkowski functional of $\Gamma_{k_{\alpha}}\left(U_{\alpha}+i \theta_{A}\right)$. (For real normed algebras the following result has been proved in [8, pages 68-69] (see also [12, page 8]) and for $k$-seminormed algebras with $k \in(0,1]$ in [7, pages 183-184]).

THEOREM 3.1. Let $(A, \tau)$ be a real locally pseudoconvex algebra, let $\left\{p_{\alpha}, \alpha \in\right.$ A\} be a family of $k_{\alpha}$-homogeneous seminorms on $A$ (with $k_{\alpha} \in(0,1]$ for each $\alpha \in A$ ), which defines the topology $\tau$ on $A$, and let $U_{\alpha}=\left\{a \in A: p_{\alpha}(a)<1\right\}$.

Then the following statements are true for each $\alpha \in \mathscr{A}$ :

(a) $q_{\alpha}$ is a $k_{\alpha}$-homogeneous seminorm on $\tilde{A}$;

(b) $\max \left\{p_{\alpha}(a), p_{\alpha}(b)\right\} \leqslant q_{\alpha}(a+i b) \leqslant 2 \max \left\{p_{\alpha}(a), p_{\alpha}(b)\right\}$ for each $a, b \in A$; 
(c) $q_{\alpha}\left(a+i \theta_{A}\right)=p_{\alpha}(a)$ for each $a \in A$;

(d) $\Gamma_{k_{\alpha}}\left(U_{\alpha}+i \theta_{A}\right)=\left\{a+i b \in \tilde{A}: q_{\alpha}(a+i b)<1\right\}$.

Proof. (a) Let $\alpha \in \mathscr{A},(a+i b) \in \tilde{A} \backslash\left\{\theta_{\tilde{A}}\right\}$, and $\mu_{\alpha}^{k_{\alpha}}>\max \left\{p_{\alpha}(a), p_{\alpha}(b)\right\}$. Then $a / \mu_{\alpha}, b / \mu_{\alpha} \in U_{\alpha}$. Since

$$
\begin{gathered}
2^{-1 / k_{\alpha}}\left(\frac{a}{\mu_{\alpha}}+i \frac{b}{\mu_{\alpha}}\right)=2^{-1 / k_{\alpha}}\left(\frac{a}{\mu_{\alpha}}+i \theta_{A}\right)+i 2^{-1 / k_{\alpha}}\left(\frac{b}{\mu_{\alpha}}+i \theta_{A}\right), \\
\left|2^{-1 / k_{\alpha}}\right|^{k_{\alpha}}+\left|i 2^{-1 / k_{\alpha}}\right|^{k_{\alpha}}=1,
\end{gathered}
$$

then

$$
(a+i b) \in 2^{1 / k_{\alpha}} \mu_{\alpha} \Gamma_{k_{\alpha}}\left(U_{\alpha}+i \theta_{A}\right) .
$$

Hence $(a+i b) \in \lambda_{\alpha} \Gamma_{k_{\alpha}}\left(U_{\alpha}+i \theta_{A}\right)$ for each $\alpha \in \mathscr{A}$ if $\left|\lambda_{\alpha}\right| \geqslant 2^{1 / k_{\alpha}} \mu_{\alpha}$. It means that the set $\Gamma_{k_{\alpha}}\left(U_{\alpha}+i \theta_{A}\right)$ is absorbing. Consequently (see [7, Proposition 4.1.10]), $q_{\alpha}$ is a $k_{\alpha}$-homogeneous seminorm on $\tilde{A}$.

(b) Let again $(a+i b) \in \tilde{A} \backslash\left\{\theta_{\tilde{A}}\right\}$. Then from (3.3), it follows that $q_{\alpha}(a+i b) \leqslant$ $2 \mu_{\alpha}^{k_{\alpha}}$. Since this inequality is valid for each $\mu_{\alpha}^{k_{\alpha}}>\max \left\{p_{\alpha}(a), p_{\alpha}(b)\right\}$, then

$$
q_{\alpha}(a+i b) \leqslant 2 \max \left\{p_{\alpha}(a), p_{\alpha}(b)\right\} .
$$

Let now $a+i b \in \Gamma_{k_{\alpha}}\left(U_{\alpha}+i \theta_{A}\right)$. Then

$$
a+i b=\sum_{k=1}^{n}\left(\lambda_{k}+i \mu_{k}\right)\left(a_{k}+i \theta_{A}\right)=\sum_{k=1}^{n} \lambda_{k} a_{k}+i \sum_{k=1}^{n} \mu_{k} a_{k}
$$

for some $a_{1}, \ldots, a_{n} \in U_{\alpha}$ and real numbers $\lambda_{1}, \ldots, \lambda_{n}$ and $\mu_{1}, \ldots, \mu_{n}$ such that

$$
\sum_{k=1}^{n}\left|\lambda_{k}+i \mu_{k}\right|^{k_{\alpha}} \leqslant 1
$$

Since $\left|\lambda_{k}\right| \leqslant\left|\lambda_{k}+i \mu_{k}\right|$ and $\left|\mu_{k}\right| \leqslant\left|\lambda_{k}+i \mu_{k}\right|$ for each $k \in\{1, \ldots, n\}$, then

$$
a=\sum_{k=1}^{n} \lambda_{k} a_{k}, \quad b=\sum_{k=1}^{n} \mu_{k} a_{k}
$$

belong to $\Gamma_{k_{\alpha}}\left(U_{\alpha}\right)=U_{\alpha}$.

Let now $\varepsilon>0$ and

$$
\mu_{\alpha}>\left(\frac{1}{q_{\alpha}(a+i b)+\varepsilon}\right)^{1 / k_{\alpha}} .
$$

Then from $\mu_{\alpha}(a+i b) \in \Gamma_{k_{\alpha}}\left(U_{\alpha}+i \theta_{A}\right)$ follows that $\mu_{\alpha} a, \mu_{\alpha} b \in U_{\alpha}$ or $p_{\alpha}\left(\mu_{\alpha} a\right)<1$ and $p_{\alpha}\left(\mu_{\alpha} b\right)<1$. Therefore

$$
\max \left\{p_{\alpha}(a), p_{\alpha}(b)\right\}<\mu_{\alpha}^{-k_{\alpha}}<q_{\alpha}(+i b)+\varepsilon .
$$


Since $\varepsilon$ is arbitrary, then from (3.9) follows that $\max \left\{p_{\alpha}(a), p_{\alpha}(b)\right\} \leqslant q_{\alpha}(a+i b)$ for each $a, b \in A$. Taking this and inequality (3.4) into account, it is clear that statement (b) holds.

(c) Let $a \in A, \alpha \in \mathcal{A}$, and $\rho^{k_{\alpha}}>q_{\alpha}\left(a+i \theta_{A}\right)$. Then from

$$
\left(\frac{a}{\rho}+i \theta_{A}\right) \in \Gamma_{k_{\alpha}}\left(U_{\alpha}+i \theta_{A}\right)
$$

it follows that $a \in \rho U_{\alpha}$ or $p_{\alpha}(a)<\rho^{k_{\alpha}}$. It means that the set of numbers $\rho^{k_{\alpha}}$ for which $\rho^{k_{\alpha}}>q_{\alpha}\left(a+i \theta_{A}\right)$ is bounded below by $p_{\alpha}(a)$. Therefore $p_{\alpha}(a) \leqslant$ $q_{\alpha}\left(a+i \theta_{A}\right)$.

Let now $\rho^{k_{\alpha}}>p_{\alpha}(a)$. Then $a \in \rho U_{\alpha}$ and from

$$
\left(\frac{a}{\rho}+i \theta_{A}\right) \in \Gamma_{k_{\alpha}}\left(U_{\alpha}+i \theta_{A}\right)
$$

it follows that $q_{\alpha}\left(a+i \theta_{A}\right)<\rho^{k_{\alpha}}$. Hence $q_{\alpha}\left(a+i \theta_{A}\right) \leqslant p_{\alpha}(a)$. Thus $q_{\alpha}(a+$ $\left.i \theta_{A}\right)=p_{\alpha}(a)$ for each $a \in A$ and $\alpha \in \mathcal{A}$.

(d) It is clear that the set $\left\{a+i b \in \tilde{A}: q_{\alpha}(a+i b)<1\right\} \subset \Gamma_{k_{\alpha}}\left(U_{\alpha}+i \theta_{A}\right)$. Let now $a+i b \in \Gamma_{k_{\alpha}}\left(U_{\alpha}+i \theta_{A}\right)$. Then

$$
a+i b=\sum_{k=1}^{n}\left(\lambda_{k}+i \mu_{k}\right)\left(a_{k}+i \theta_{A}\right)
$$

for some $a_{1}, \ldots, a_{n} \in U_{\alpha}$ and real numbers $\lambda_{1}, \ldots, \lambda_{n}$ and $\mu_{1}, \ldots, \mu_{n}$ such that

$$
\sum_{k=1}^{n}\left|\lambda_{k}+i \mu_{k}\right|^{k_{\alpha}} \leqslant 1
$$

Since $p_{\alpha}\left(a_{k}\right)<1$ for each $k \in\{1, \ldots, n\}$, we can choose $\varepsilon_{\alpha}>0$ so that

$$
\max \left\{p_{\alpha}\left(a_{1}\right), \ldots, p_{\alpha}\left(a_{n}\right)\right\}<\varepsilon_{\alpha}^{k_{\alpha}}<1
$$

Then $a_{k} \in \varepsilon_{\alpha} U_{\alpha}$ for each $\alpha \in \mathscr{A}$ and each $k \in\{1, \ldots, n\}$. Therefore

$$
\frac{a+i b}{\varepsilon_{\alpha}} \in \sum_{k=1}^{n}\left(\lambda_{k}+i \mu_{k}\right)\left(\frac{a_{k}}{\varepsilon_{\alpha}}+i \theta_{A}\right) \in \Gamma_{k_{\alpha}}\left(U_{\alpha}+i \theta_{A}\right) .
$$

Hence

$$
(a+i b) \in \varepsilon_{\alpha} \Gamma_{k_{\alpha}}\left(U_{\alpha}+i \theta_{A}\right)
$$

or $q_{\alpha}(a+i b) \leqslant \varepsilon_{\alpha}^{k_{\alpha}}<1$. It means that statement (d) holds.

COROLLARY 3.2. If $(A, \tau)$ is a real locally pseudoconvex Fréchet algebra, then $(\tilde{A}, \tilde{\tau})$ is a complex locally pseudoconvex Fréchet algebra. 
Proof. Let $(A, \tau)$ be a real locally pseudoconvex Fréchet algebra and let $\left\{p_{n}, n \in \mathbb{N}\right\}$ be a countable family of $k_{n}$-homogeneous seminorms (with $k_{n} \in$ $(0,1]$ for each $n \in \mathbb{N})$, which defines the topology $\tau$ on $A$. Then $\left\{q_{n}: n \in \mathbb{N}\right\}$ defines on $\tilde{A}$ a metrizable locally pseudoconvex topology $\tilde{\tau}$ (see Theorem 3.1). If $\left(a_{n}+i b_{n}\right)$ is a Cauchy sequence in $(\tilde{A}, \tilde{\tau})$, then $\left(a_{n}\right)$ and $\left(b_{n}\right)$ are Cauchy sequences in $(A, \tau)$ by Theorem 3.1(b). Because $(A, \tau)$ is complete, then $\left(a_{n}\right)$ converges to $a_{0} \in A$ and $\left(b_{n}\right)$ converges to $b_{0} \in A$. Hence $\left(a_{n}+i b_{n}\right)$ converges in $(\tilde{A}, \tilde{\tau})$ to $a_{0}+i b_{0} \in \tilde{A}$ by the same inequality (b). Thus $(\tilde{A}, \tilde{\tau})$ is a complex locally pseudoconvex Fréchet algebra.

THEOREM 3.3. Let $(A, \tau)$ be a real locally A-pseudoconvex (locally m-

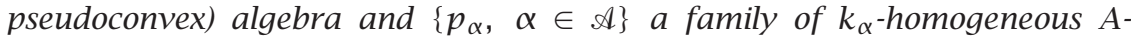
multiplicative (resp., submultiplicative) seminorms on $A$ (with $k_{\alpha} \in(0,1]$ for each $\alpha \in \mathscr{A})$, which defines the topology $\tau$ on $A$. Then $(\tilde{A}, \tilde{\tau})$ is a complex locally A-pseudoconvex (resp., locally m-pseudoconvex) algebra. (Here $\tilde{\tau}$ denotes the topology on $\tilde{A}$ defined by the system $\left\{q_{\alpha}: \alpha \in \mathscr{A}\right\}$.)

Proof. Let $p_{\alpha}$ be an $A$-multiplicative seminorm on $A$. Then for each fixed element $a_{0} \in A$, there are numbers $M_{\alpha}\left(a_{0}\right)>0$ and $N_{\alpha}\left(a_{0}\right)>0$ such that

$$
p_{\alpha}\left(a_{0} a\right) \leqslant M_{\alpha}\left(a_{0}\right) p_{\alpha}(a), \quad p_{\alpha}\left(a a_{0}\right) \leqslant N_{\alpha}\left(a_{0}\right) p_{\alpha}(a),
$$

for each $a \in A$. If $a_{0}+i b_{0}$ is a fixed element and $a+i b$ an arbitrary element of $\tilde{A}$, then

$$
\begin{aligned}
q_{\alpha}\left(\left(a_{0}+i b_{0}\right)(a+i b)\right) & =q_{\alpha}\left(\left(a_{0} a-b_{0} b\right)+i\left(a_{0} b+b_{0} a\right)\right) \\
& \leqslant 2 \max \left\{p_{\alpha}\left(a_{0} a-b_{0} b\right), p_{\alpha}\left(a_{0} b+b_{0} a\right)\right\}
\end{aligned}
$$

by Theorem 3.1(b). If now $p_{\alpha}\left(a_{0} a-b_{0} b\right) \geqslant p_{\alpha}\left(a_{0} b+b_{0} a\right)$, then

$$
\begin{aligned}
\max \left\{p_{\alpha}\right. & \left.\left(a_{0} a-b_{0} b\right), p_{\alpha}\left(a_{0} b+b_{0} a\right)\right\} \\
& =p_{\alpha}\left(a_{0} a-b_{0} b\right) \\
& \leqslant M_{\alpha}\left(a_{0}\right) p_{\alpha}(a)+M_{\alpha}\left(b_{0}\right) p_{\alpha}(b) \\
& \leqslant \max \left\{p_{\alpha}(a), p_{\alpha}(b)\right\}\left(M_{\alpha}\left(a_{0}\right)+M_{\alpha}\left(b_{0}\right)\right) \\
& \leqslant \frac{1}{2} M_{\alpha}\left(a_{0}, b_{0}\right) q_{\alpha}(a+i b)
\end{aligned}
$$

by Theorem 3.1(b) (here $\left.M_{\alpha}\left(a_{0}, b_{0}\right)=2\left(M_{\alpha}\left(a_{0}\right)+M_{\alpha}\left(b_{0}\right)\right)\right)$. Hence

$$
q_{\alpha}\left(\left(a_{0}+i b_{0}\right)(a+i b)\right) \leqslant M_{\alpha}\left(a_{0}, b_{0}\right) q_{\alpha}(a+i b)
$$

for each $a+i b \in \tilde{A}$.

The proof for the case when $p_{\alpha}\left(a_{0} a-b_{0} b\right)<p_{\alpha}\left(a_{0} b+b_{0} a\right)$ is similar. Thus inequality (3.20) holds for both cases. In the same way, it is easy to show that the inequality

$$
q_{\alpha}\left((a+i b)\left(a_{0}+i b_{0}\right)\right) \leqslant N_{\alpha}\left(a_{0}, b_{0}\right) q_{\alpha}(a+i b)
$$


holds for each $a+i b \in \tilde{A}$. Consequently, $(\tilde{A}, \tilde{\tau})$ is a complex locally $A$-pseudoconvex algebra.

Let now $p_{\alpha}$ be a submultiplicative seminorm on $A$. Then $p_{\alpha}(a b) \leqslant p_{\alpha}(a) p_{\alpha}(b)$ for each $a, b \in A$. If $a+i b, a^{\prime}+i b^{\prime} \in \tilde{A}$, then

$$
q_{\alpha}\left((a+i b)\left(a^{\prime}+i b^{\prime}\right)\right) \leqslant 2 \max \left\{p_{\alpha}\left(a a^{\prime}-b b^{\prime}\right), p_{\alpha}\left(a b^{\prime}+b a^{\prime}\right)\right\}
$$

by Theorem 3.1(b). If now $p_{\alpha}\left(a a^{\prime}-b b^{\prime}\right) \geqslant p_{\alpha}\left(a b^{\prime}+b a^{\prime}\right)$, then

$$
\begin{aligned}
\max & \left\{p_{\alpha}\left(a a^{\prime}-b b^{\prime}\right), p_{\alpha}\left(a b^{\prime}+b a^{\prime}\right)\right\} \\
& =p_{\alpha}\left(a a^{\prime}-b b^{\prime}\right) \leqslant p_{\alpha}(a) p_{\alpha}\left(a^{\prime}\right)+p_{\alpha}(b) p_{\alpha}\left(b^{\prime}\right) \\
& \leqslant 2 \max \left\{p_{\alpha}(a), p_{\alpha}(b)\right\} \max \left\{p_{\alpha}\left(a^{\prime}\right), p_{\alpha}\left(b^{\prime}\right)\right\} \\
& \leqslant 2 q_{\alpha}(a+i b) q_{\alpha}\left(a^{\prime}+i b^{\prime}\right)
\end{aligned}
$$

by Theorem 3.1(b). Hence

$$
q_{\alpha}\left((a+i b)\left(a^{\prime}+i b^{\prime}\right)\right) \leqslant 4 q_{\alpha}(a+i b) q_{\alpha}\left(a^{\prime}+i b^{\prime}\right) .
$$

Putting $r_{\alpha}=4 q_{\alpha}$ for each $\alpha \in \mathscr{A}$, we see that

$$
r_{\alpha}\left((a+i b)\left(a^{\prime}+i b^{\prime}\right)\right) \leqslant r_{\alpha}(a+i b) r_{\alpha}\left(a^{\prime}+i b^{\prime}\right)
$$

for each $a+i b, a^{\prime}+i b^{\prime} \in \tilde{A}$.

The proof for the case when $p_{\alpha}\left(a a^{\prime}-b b^{\prime}\right)<p_{\alpha}\left(a b^{\prime}+b a^{\prime}\right)$ is similar. Hence inequality (3.25) holds for both cases. Since the families $\left\{q_{\alpha}: \alpha \in \mathscr{A}\right\}$ and $\left\{r_{\alpha}: \alpha \in \mathscr{A}\right\}$ define on $\tilde{A}$ the same topology, then $(\tilde{A}, \tilde{\tau})$ is a complex locally $m$-pseudoconvex algebra.

4. Complexification of real exponentially galbed algebras. Next, we will show that the complexification $(\tilde{A}, \tilde{\tau})$ of $(A, \tau)$ is a complex exponentially galbed algebra if $(A, \tau)$ is a real exponentially galbed algebra, and all elements of $(\tilde{A}, \tilde{\tau})$ are bounded in $(\tilde{A}, \tilde{\tau})$ if $(A, \tau)$ is a commutative exponentially galbed algebra in which all elements are bounded and the multiplication in $(A, \tau)$ is jointly continuous.

THEOREM 4.1. Let $(A, \tau)$ be a real exponentially galbed algebra (commutative real exponentially galbed algebra with jointly continuous multiplication and bounded elements). Then $(\tilde{A}, \tilde{\tau})$ is a complex exponentially galbed algebra (resp., commutative complex exponentially galbed algebra with bounded elements).

Proof. Let $(A, \tau)$ be a real exponentially galbed algebra and $\tilde{O}$ a neighborhood of zero in $(\tilde{A}, \tilde{\tau})$. Then there are a neighborhood $O$ of zero of $(A, \tau)$ such that $O+i O \subset \tilde{O}$ and another neighborhood $U$ of zero of $(A, \tau)$ such that

$$
\left\{\sum_{k=0}^{n} \frac{a_{k}}{2^{k}}: a_{0}, \ldots, a_{n} \in U\right\} \subset O
$$


for each $n \in \mathbb{N}$. Since $U+i U$ is a neighborhood of zero in $(\tilde{A}, \tilde{\tau})$ and

$$
\left\{\sum_{k=0}^{n} \frac{a_{k}+i b_{k}}{2^{k}}: a_{0}+i b_{0}, \ldots, a_{n}+i b_{n} \in U+i U\right\} \subset O+i O \subset \tilde{O}
$$

for each $n \in \mathbb{N}$, then $(\tilde{A}, \tilde{\tau})$ is a complex exponentially galbed algebra.

Let now $(A, \tau)$ be a commutative real exponentially galbed algebra with jointly continuous multiplication and bounded elements, $\tilde{O}$ an arbitrary neighborhood of zero of $(\tilde{A}, \tilde{\tau})$, and $a+i b \in \tilde{A}$ an arbitrary element. Then there are a neighborhood $O$ of zero of $(A, \tau)$ such that $O+i O \subset \tilde{O}$ and $\lambda_{a}, \lambda_{b} \in \mathbb{C} \backslash\{0\}$ and the sets

$$
\left\{\left(\frac{a}{\lambda_{a}}\right)^{n}: n \in \mathbb{N}\right\}, \quad\left\{\left(\frac{b}{\lambda_{b}}\right)^{n}: n \in \mathbb{N}\right\}
$$

are bounded in $(A, \tau)$. The neighborhood $O$ defines now a balanced neighborhood $U$ of zero of $(A, \tau)$ such that (4.2) holds and $U$ defines a balanced neighborhood $V$ of zero of $(A, \tau)$ such that $V V \subset U$ (because the multiplication in $(A, \tau)$ is jointly continuous). Now there are numbers $\mu_{a}, \mu_{b}>0$ such that

$$
\left(\frac{a}{\left|\lambda_{a}\right|}\right)^{n} \in \mu_{a} V, \quad\left(\frac{b}{\left|\lambda_{b}\right|}\right)^{n} \in \mu_{b} V
$$

for each $n \in \mathbb{N}$. Let $\kappa=4\left(\left|\lambda_{a}\right|+\left|\lambda_{b}\right|\right)$. Since $a+i b=\left(a+i \theta_{A}\right)+i\left(b+i \theta_{A}\right)$, then

$$
\begin{aligned}
\left(\frac{a+i b}{\kappa}\right)^{n} & =\sum_{k=0}^{n}\left(\begin{array}{l}
n \\
k
\end{array}\right)\left(\left(\frac{a}{\kappa}\right)^{k}+i \theta_{A}\right) i^{n-k}\left(\left(\frac{b}{\kappa}\right)^{n-k}+i \theta_{A}\right) \\
& =\mu_{a} \mu_{b} \sum_{k=0}^{n} \frac{\tilde{x}_{k}}{2^{k}}
\end{aligned}
$$

for each $n \in \mathbb{N}$, where

$$
\begin{gathered}
\tilde{x}_{k}=\varrho_{n k} \frac{1}{\mu_{a} \mu_{b}}\left(\left(\frac{a}{\left|\lambda_{a}\right|}\right)^{k}\left(\frac{b}{\left|\lambda_{b}\right|}\right)^{n-k}+i \theta_{A}\right), \\
\varrho_{n k}=2^{k} i^{n-k}\left(\begin{array}{l}
n \\
k
\end{array}\right)\left(\frac{\left|\lambda_{a}\right|}{\kappa}\right)^{k}\left(\frac{\left|\lambda_{b}\right|}{\kappa}\right)^{n-k},
\end{gathered}
$$

for each $k \leqslant n$. Herewith

$$
\begin{gathered}
\left|\varrho_{n k}\right|=\frac{2^{k}}{\kappa^{n}}\left(\begin{array}{c}
n \\
k
\end{array}\right)\left|\lambda_{a}\right|^{k}\left|\lambda_{b}\right|^{n-k} \leqslant \frac{2^{n}}{\kappa^{n}}\left(\left|\lambda_{a}\right|+\left|\lambda_{b}\right|\right)^{n} \leqslant\left(\frac{1}{2}\right)^{n}<1, \\
\left(\frac{a}{\left|\lambda_{a}\right|}\right)^{k}\left(\frac{b}{\left|\lambda_{b}\right|}\right)^{n-k}+i \theta_{A} \in \mu_{a} \mu_{b} V V+i \theta_{A} \subset \mu_{a} \mu_{b}(U+i U) .
\end{gathered}
$$


Since $U$ is a balanced set, then $\tilde{x}_{k} \in U+i U$ for each $k \in\{0, \ldots, n\}$. Hence

$$
\left(\frac{a+i b}{\kappa}\right)^{n} \in \mu_{a} \mu_{b}(O+i O) \subset \mu_{a} \mu_{b} \tilde{O}
$$

by (4.2) for each $n \in \mathbb{N}$. It means that $a+i b$ is bounded in $(\tilde{A}, \tilde{\tau})$. Consequently, $(\tilde{A}, \tilde{\tau})$ is a commutative complex exponentially galbed algebra with bounded elements.

5. Real Gel'fand-Mazur division algebras. To describe main classes of real Gel'fand-Mazur division algebras, we first describe these real topological division algebras $(A, \tau)$ for which the complexification $(\tilde{A}, \tilde{\tau})$ of $(A, \tau)$ is a complex Gel'fand-Mazur division algebra.

Proposition 5.1. If $(A, \tau)$ is a commutative strictly real topological Hausdorff division algebra with continuous inversion, then the complexification $(\tilde{A}, \tilde{\tau})$ of $(A, \tau)$ is a commutative complex topological Hausdorff division algebra with continuous inversion.

Proof. Let $A$ be a commutative strictly real division algebra. Then $\tilde{A}$ is a complex division algebra (see [7, Proposition 1.6.20]). Since the underlying topological space of $(A, \tau)$ is a Hausdorff space, then $(A, \tau)$ is a $Q$-algebra. Hence $(A, \tau)$ is a commutative real Waelbroeck algebra with a unit element. Therefore $(\tilde{A}, \tilde{\tau})$ is a commutative Waelbroeck algebra (see [7, Proposition 3.6.31] or [17, proposition on page 237]). Thus, $(\tilde{A}, \tilde{\tau})$ is a commutative complex Hausdorff division algebra with continuous inversion.

Proposition 5.2. Let $(A, \tau)$ be a real topological algebra and $\tilde{A}$ the complexification of $A$. If the topological dual $(A, \tau) *$ of $(A, \tau)$ is nonempty, then the topological dual $(\tilde{A}, \tilde{\tau})^{*}$ of $(\tilde{A}, \tilde{\tau})$ is also nonempty.

Proof. If $\psi \in(A, \tau)^{*}$, then $\tilde{\psi}$, defined by $\tilde{\psi}(a+i b)=\psi(a)+i \psi(b)$ for each $a+i b \in \tilde{A}$, is an element of $(\tilde{A}, \tilde{\tau})^{*}$.

Proposition 5.3. Let A be a commutative strictly real (not necessarily topological) division algebra and $\tilde{A}$ the complexification of $A$. Then

$$
\operatorname{sp}_{\tilde{A}}(a+i b)=\left\{\alpha+i \beta \in \mathbb{C}: \alpha \in \operatorname{sp}_{A}(a) \text { and } \beta \in \operatorname{sp}_{A}(b)\right\} \text {. }
$$

Proof. Let $\alpha+i \beta \in \operatorname{sp}_{\tilde{A}}(a+i b)$. Since $A$ is a commutative strictly real division algebra, then $\tilde{A}$ is a commutative complex division algebra (see [7, Proposition 1.6.20]). Therefore

$$
a+i b-(\alpha+i \beta)\left(e_{A}+i \theta\right)=\left(a-\alpha e_{A}\right)+i\left(b-\beta e_{A}\right)=\theta_{A}+i \theta_{A}
$$

if and only if $\alpha \in \operatorname{sp}_{A}(a)$ and $\beta \in \operatorname{sp}_{A}(b)$.

The main result of the present paper is the following theorem. 
THEOREM 5.4. Let $(A, \tau)$ be a commutative strictly real topological division algebra and $\tilde{A}$ the complexification of $A$. If there is a topology $\tau^{\prime}$ on $A$ such that $\left(A, \tau^{\prime}\right)$ is

(a) a locally pseudoconvex Hausdorff algebra with continuous inversion;

(b) a Hausdorff algebra with continuous inversion for which $(A, \tau)^{*}$ is nonempty;

(c) an exponentially galbed Hausdorff algebra with jointly continuous multiplication and bounded elements;

(d) a topological Hausdorff algebra for which the spectrum $\operatorname{sp}_{A}(a)$ is nonempty for each $a \in A$,

then $(A, \tau)$ and $\mathbb{R}$ are topologically isomorphic.

PROof. If $A$ is a commutative strictly real division algebra, then $\tilde{A}$ is a commutative complex division algebra (by [7, Proposition 1.6.20]). In case (a) the complexification $\left(\tilde{A}, \tilde{\tau}^{\prime}\right)$ of $\left(A, \tau^{\prime}\right)$ is a commutative complex locally pseudoconvex Hausdorff division algebra with continuous inversion (by Theorem 3.1 and Proposition 5.1); in case (b) $\left(\tilde{A}, \tilde{\tau}^{\prime}\right)$ of $\left(A, \tau^{\prime}\right)$ is a commutative complex topological Hausdorff algebra with continuous inversion for which the set $\left(\tilde{A}, \tilde{\tau}^{\prime}\right)^{*}$ is nonempty (by Propositions 5.1 and 5.2$)$; in case (c) $\left(\tilde{A}, \tilde{\tau}^{\prime}\right)$ of $\left(A, \tau^{\prime}\right)$ is a commutative complex exponentially galbed Hausdorff division algebra with bounded elements (by Theorem 4.1); and in case (d) $\left(\tilde{A}, \tilde{\tau}^{\prime}\right)$ of $\left(A, \tau^{\prime}\right)$ is such a commutative topological Hausdorff division algebra for which the spectrum $\operatorname{sp}_{\tilde{A}}(a+i b)$ is nonempty for each $a+i b \in \tilde{A}$ (by Proposition 5.3), therefore $(\tilde{A}, \tilde{\tau})$ and $\mathbb{C}$ are topologically isomorphic (see [4, Theorem 1] and [2, Proposition 1]). Hence every element $a+i b \in \tilde{A}$ is representable in the form $a+i b=\lambda e_{\tilde{A}}$ for some $\lambda \in \mathbb{C}$. It means that for each $a \in A$ there is a real number $\mu$ such that $a=\mu e_{A}$. Consequently, $A$ is an isomorphism to $\mathbb{R}$. In the same way as in complex case (see, e.g., [4, page 122]) it is easy to show that this isomorphism is a topological isomorphism because $(A, \tau)$ is a Hausdorff space.

COROLLARY 5.5. Let $A$ be a commutative strictly real division algebra. If $A$ has a topology $\tau$ such that $(A, \tau)$ is

(a) a locally pseudoconvex Hausdorff algebra with continuous inversion;

(b) a locally A-pseudoconvex (in particular, locally m-pseudoconvex) Hausdorff algebra;

(c) a locally pseudoconvex Fréchet algebra;

(d) an exponentially galbed Hausdorff algebra with jointly continuous multiplication and bounded elements;

(e) a topological Hausdorff algebra for which the spectrum $\operatorname{sp}_{A}(a)$ is nonempty for each $a \in A$,

then $(A, \tau)$ is a commutative real Gel'fand-Mazur division algebra.

Proof. It is easy to see that $(A, \tau)$ is a commutative real Gel'fand-Mazur division algebra (by Theorem 5.4) in cases (a), (d), and (e). Since the inversion 
is continuous in every locally $m$-pseudoconvex algebra and every locally $A$ pseudoconvex Hausdorff algebra with a unit element having a topology $\tau^{\prime}$ such that $\left(A, \tau^{\prime}\right)$ is a locally $m$-pseudoconvex Hausdorff algebra (see [5, Lemma 2.2]), then $(A, \tau)$ is a commutative real Gel'fand-Mazur division algebra in case (b) by (a) and Theorem 5.4 .

Let now $(A, \tau)$ be a commutative strictly real locally pseudoconvex Fréchet division algebra. Then $(A, \tau)$ is a commutative strictly real locally pseudoconvex Fréchet $Q$-algebra by Corollary 3.2. Therefore the inversion in $(A, \tau)$ is continuous (see [15, Corollary 7.6]). Hence $(A, \tau)$ is also a commutative real Gel'fand-Mazur division algebra by Theorem 5.4 .

ACKNOWLedgment. This research was supported in part by an Estonian Science Foundation Grant 4514.

\section{REFERENCES}

[1] M. Abel, Inductive limits of Gel'fand-Mazur algebras, submitted to J. Austral. Math. Soc.

[2] _ Survey of results on Gel'fand-Mazur algebras, Proc. of the Intern. Conf. on Topological Algebras and Applications (Rabat 2000), to appear.

[3] _ On the Gel'fand-Mazur theorem for exponentially galbed algebras, Tartu Riikl. Ül. Toimetised (1990), no. 899, 65-70.

[4] Gel'fand-Mazur algebras, Topological Vector Spaces, Algebras and Related Areas (Hamilton, ON, 1994), Pitman Res. Notes Math. Ser., vol. 316, Longman Scientific \& Technical, Harlow, 1994, pp. 116-129.

[5] M. Abel and A. Kokk, Locally pseudoconvex Gel'fand-Mazur algebras, Eesti NSV Tead. Akad. Toimetised Füüs.-Mat. 37 (1988), no. 4, 377-386 (Russian).

[6] G. R. Allan, A spectral theory for locally convex algebras, Proc. London Math. Soc. (3) 15 (1965), 399-421.

[7] V. K. Balachandran, Topological Algebras, North-Holland Mathematics Studies, vol. 185, North-Holland Publishing, Amsterdam, 2000, reprint of the 1999 original.

[8] F. F. Bonsall and J. Duncan, Complete Normed Algebras, Ergebnisse der Mathematik und ihrer Grenzgebiete, vol. 80, Springer-Verlag, New York, 1973.

[9] A. Guichardet, Special Topics in Topological Algebras, Gordon and Breach Science Publishers, New York, 1968.

[10] A. Mallios, Topological Algebras. Selected Topics, North-Holland Mathematics Studies, vol. 124, North-Holland Publishing, Amsterdam, 1986.

[11] M. A. Nămark, Normed Algebras, Wolters-Noordhoff Publishing, Groningen, 1972.

[12] C. E. Rickart, General Theory of Banach Algebras, The University Series in Higher Mathematics, D. van Nostrand, Princeton, 1960.

[13] Ph. Turpin, Espaces et opérateurs exponentiellement galbés, Séminaire Pierre Lelong (Analyse), Année 1973-1974, Lecture Notes in Math., vol. 474, Springer, Berlin, 1975, pp. 48-62 (French).

[14] L. Waelbroeck, Topological Vector Spaces and Algebras, Lecture Notes in Mathematics, vol. 230, Springer-Verlag, Berlin, 1971.

[15] W. Żelazko, Metric generalizations of Banach algebras, Rozprawy Mat. 47 (1965), $1-70$. 
[16] _ Selected Topics in Topological Algebras, Lecture Notes Series, no. 31, Matematisk Institut, Aarhus Universitet, Aarhus, 1971.

[17] _ On m-convexity of commutative real Waelbroeck algebras, Comment. Math. Prace Mat. 40 (2000), 235-240.

Mati Abel: Institute of Pure Mathematics, University of Tartu, 2 J. Liivi Street, 50409 Tartu, Estonia

E-mail address: abe1@math. ut. ee

Olga Panova: Institute of Pure Mathematics, University of Tartu, 2 J. Liivi Street, 50409 Tartu, Estonia

E-mail address: olpanova@math.ut.ee 


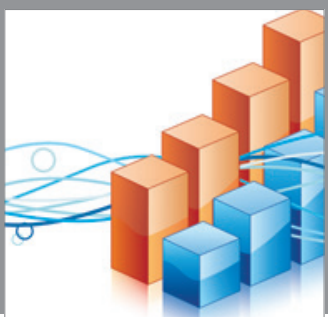

Advances in

Operations Research

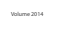

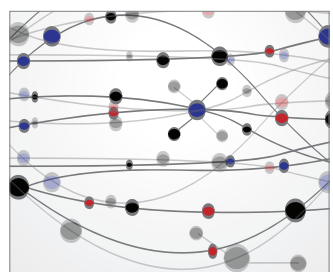

\section{The Scientific} World Journal
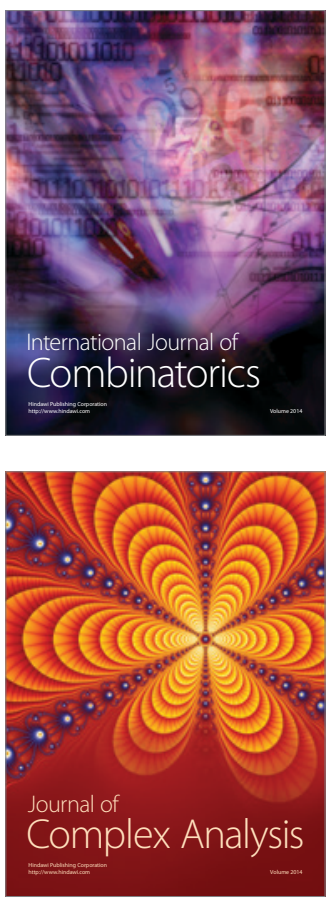

International Journal of

Mathematics and

Mathematical

Sciences
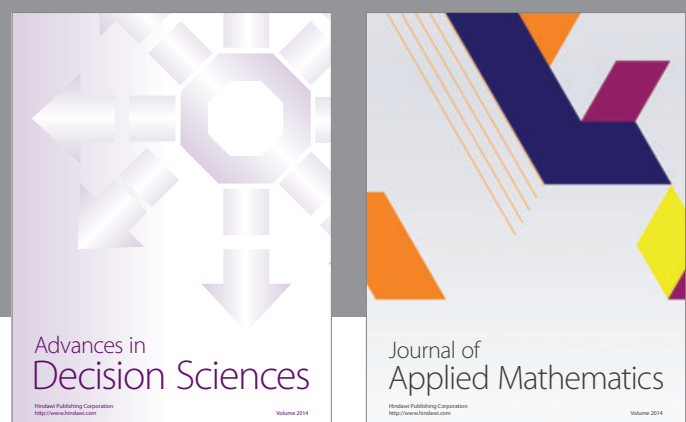

Journal of

Applied Mathematics
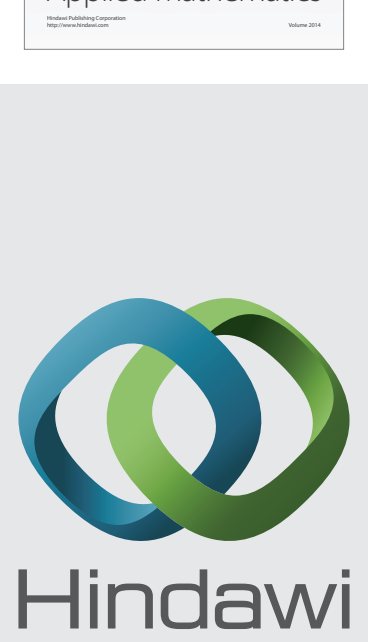

Submit your manuscripts at http://www.hindawi.com
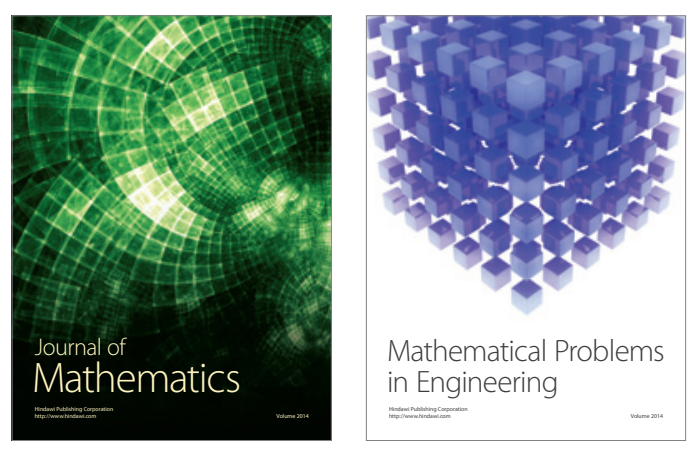

Mathematical Problems in Engineering
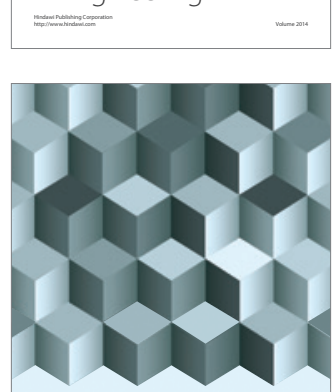

Journal of

Function Spaces
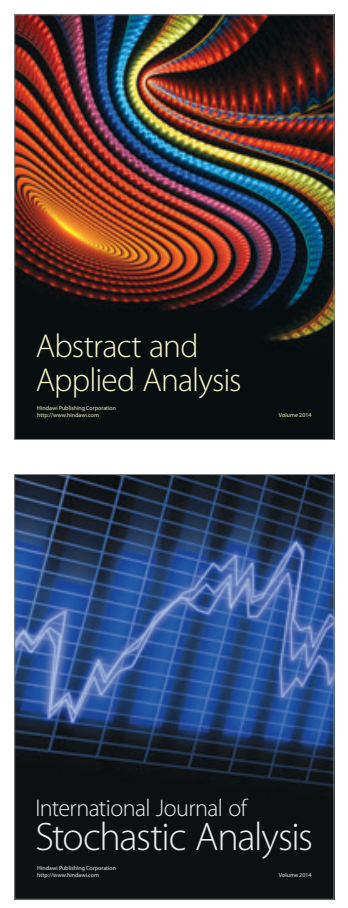

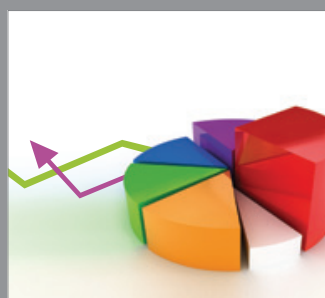

ournal of

Probability and Statistics

Promensencen
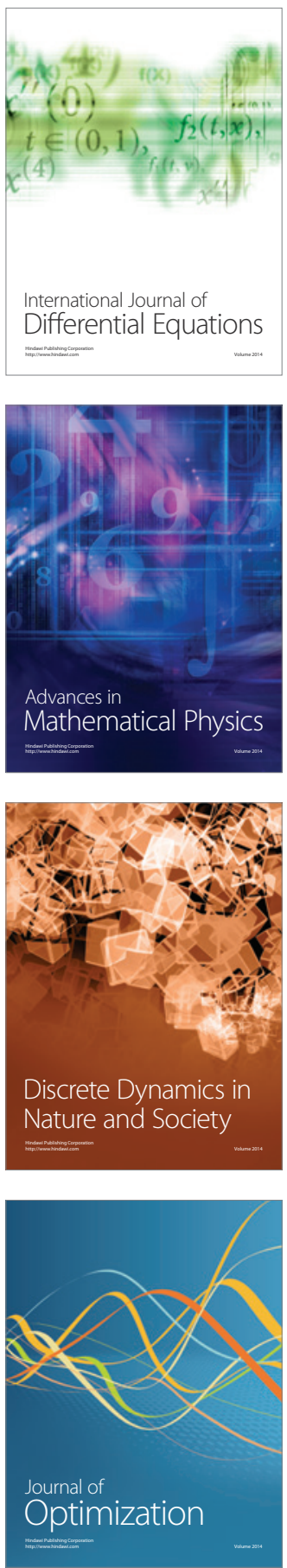